\title{
Protocol
}

\section{Defining the older patient population that require, but do not undergo emergency laparotomy: an observational cohort study protocol}

\author{
Nicola Reeves ${ }^{1}$, Susan Chandler ${ }^{2}$, Elizabeth McLennan ${ }^{3}$, Angeline Price ${ }^{4}$, Jemma Boyle ${ }^{5}$, \\ Stephen Knight ${ }^{6}$, Lyndsay Pearce $^{7}$, Susan Moug ${ }^{8 *}$
}

\author{
${ }^{1}$ University Hospital of Wales, Cardiff, UK \\ ${ }^{2}$ Singleton Hospital, Swansea, UK \\ ${ }^{3}$ Royal Glasgow Infirmary, Glasgow, UK \\ ${ }^{4}$ Salford Royal NHS Foundation Trust, UK \\ ${ }^{5}$ RCS England, London School of Hygiene and Tropical Medicine, UK \\ ${ }^{6}$ Centre for Medical Informatics, Usher Institute, University of Edinburgh, UK \\ ${ }^{7}$ Salford Royal NHS Foundation Trust, Stott Lane, Salford, Manchester, UK \\ ${ }^{8}$ Department of General Surgery, Royal Alexandra Hospital, Corsebar Road, Paisley, UK
}

Received: 27 January 2021

Revised: 25 February 2021

Accepted: 27 February 2021

\author{
*Correspondence: \\ Dr. Susan Moug, \\ E-mail: susan.moug@ggc.scot.nhs.uk
}

Copyright: (c) the author(s), publisher and licensee Medip Academy. This is an open-access article distributed under the terms of the Creative Commons Attribution Non-Commercial License, which permits unrestricted non-commercial use, distribution, and reproduction in any medium, provided the original work is properly cited.

\begin{abstract}
Background: Despite older adults (65 years and above) accounting for almost half of emergency laparotomies and an ageing population, there remains a paucity of research in the older adult emergency surgery population. One key clinical area that requires urgent assessment is the older patient who presents with acute abdominal pathology treatable by laparotomy, but who does not undergo surgery (NoLAP).

Methods: This multicentre prospective cohort study [defining the denominator: emergency laparotomy and frailty study 2 (ELF2)] will recruit consecutive older adults that require but do not undergo emergency laparotomy (NoLAP). We will recruit from 47 national health service hospitals over a 3-month timeframe. The same criteria as NELA for inclusion and exclusion will be applied. The primary aim is 90-day mortality. Secondary aims include characterisation of the NoLAP group, frailty and sarcopenia with comparison to those older adults that have undergone emergency laparotomy (ELAP). Decision-making will also be explored. Assuming a NoLAP rate of $32 \%$ and $10 \%$ dropout, a minimum of 700 patients are required for $95 \%$ power (alpha $=0.05$ ).

Conclusions: The UK national emergency laparotomy audit has provided vital information on those patients undergoing emergency laparotomy and driven standards in operative and perioperative care. However, little is known of outcomes in those patients who do not undergo emergency laparotomy. Improved understanding of this NoLAP population would aid shared decision-making and improve standards for this otherwise poorly understood vulnerable patient group.
\end{abstract}

Trial registration: This study is registered online at www.clinicaltrial.gov (Reg number: ISRCTN14556210).

Keywords: Laparotomy, Older patient, Outcomes, Frailty

\section{INTRODUCTION}

The work of the national emergency laparotomy audit (NELA) has significantly improved the U.K. landscape for acute surgical units performing emergency laparotomies (ELAP). ${ }^{1}$ By characterising the ELAP population and publishing the short and long-term outcomes, acute surgical units have been facilitated to 
identify areas of clinical priority and perform quality improvement interventions that have led to improved patient outcomes.

One such example is the emergency laparotomy and frailty study (ELF). ${ }^{2}$ Frailty, a relatively new concept within the medical world, is defined as 'a medical syndrome with multiple causes and contributors that is characterised by diminished strength, endurance, and reduced physiologic function that increases an individual's vulnerability for developing increased dependency and/or death' ${ }^{3}$ ELF assessed the influence of frailty on older adults (65 years and above) undergoing ELAP finding that, independent of age, as the frailty score increased the risk of 90-mortality did too. Similar findings were reported with 30-day mortality, postoperative complications, length of critical care and overall hospital stay. With increasing frailty also predicting increased care level upon discharge, the authors concluded that frailty scoring provided much needed new knowledge to inform and guide both surgeons and older adults as they consider the implications of undergoing emergency laparotomy.

In contrast, there is one surgically important area that remains unknown: older patients that require, but do not undergo emergency laparotomy (NoLAP). Similar to emergency laparotomy patients before NELA started, the NoLAP population remain uncharacterised with unknown outcomes. It is not known how many patients constitute this group, what characteristics they have, what the reasons are for not undergoing surgery and what their short and long-term outcomes are.

\section{Primary aims}

The primary outcome is 90-day mortality in older patients presenting with acute abdominal pathology potentially treatable by emergency laparotomy who do not undergo surgery (NoLAP).

\section{Secondary aims}

The secondary aim is to characterise the older patient population, with additional data points including frailty scoring, sarcopenia calculation and longer-term outcomes.

\section{METHODS}

\section{Study design}

A UK multicentre prospective observational cohort study.

\section{Study setting}

Any hospital in the UK that provides emergency general surgical services are eligible to participate with 40 having expressed interest. The study has been advertised on social media (@Elf_Study) and presented at the national research collaborative meeting 2019 (@NRCM). The study will be conducted by trainees via established surgical and geriatric trainee-led research collaboratives and guided by OPSOC. The ELF2 steering committee (see below) developed the protocol, obtained ethical approval, and will provide central organisation and coordination of the study. Swansea trials unit (STU) will provide support with administration, data collection and data management (https://swanseatrialsunit.org/).

\section{ELF2 steering committee}

The steering committee comprises of consultant general surgeons and geriatricians previously involved in the ELF study. ${ }^{2}$ The main collaborative groups that will work on this study are the Welsh Barbers, Scottish surgical research group (SSRG) and older persons surgical outcomes collaboration (OPSOC). The steering committee is responsible for protocol design, data handling, analysis, dissemination of results preparation of manuscripts and responsible for the use of data resulting from this project.

\section{Principal investigators}

Local sites will each have a principal investigator (PI) who will be responsible for leading the local teams collecting data. The PI will be responsible for liaising with their local research and development departments to ensure study approval and that the local teamwork within local clinical governance and guidelines. The PI will be the liaison between the ELF2 steering committee and their local team, ensuring dissemination of study information and the first point of contact at each site.

\section{Inclusion criteria}

All patients 65 years or older on the date of surgical decision. Patients must have been reviewed by a general surgeon (trainee+/or consultant), but can be on any ward in a hospital (e.g., ITU, gynaecology and acute medical unit). Patients must have a surgical condition that may be treatable with an emergency laparotomy as defined by the NELA criteria (Appendix A). ${ }^{1}$

\section{Exclusion criteria}

Patients aged less than 65. Patients initially requiring ELAP, but where surgical decision was made to manage conservatively will be excluded, even if that decision is subsequently changed and ELAP is performed. Cases where radiological drainage has failed (e.g., complex diverticulitis). Surgical pathology outside NELA inclusion criteria (NELA exclusion criteria) (Appendix A). ${ }^{1}$

\section{Patient identification and data collection}

Patients will be screened by the local team against the inclusion criteria with each site developing their own 
strategies for identifying patients. The ELF 2 group has suggested highlighting to their colleagues (consultant and trainees) their participation in the ELF 2 study to ensure vigilance and accurate prospective data collection. Potential patients can be recruited from any site in the hospital including non-surgical wards e.g., ITU, gynaecology and acute medical units. Data collection will be via REDCap which is an anonymised database hosted by STU. A key sheet will be kept separately with the national health service (NHS) number.

\section{Variables collected}

Demographics, comorbidities and polypharmacy data will be recorded. The Charlson co-morbidity index will be used to record co-morbidities (a validated measure of prognostic impact of multiple chronic illnesses). ${ }^{8}$ Place of residence, own house or residential/nursing home will be recorded alongside social services support (1-4 times per day package of care) to assess pre-admission independence status. Clinical Frailty Score will be assessed by the local team, a validated score that was used in the ELF study. ${ }^{9}$ Briefly this validated score ranks from 1-7 with $\geq 5$ classed as frail. The adjusted NELA risk prediction score will be recorded to indicate the preoperative risk. ${ }^{10}$ Data will be collected from patient case notes on clinical and discharge outcomes, 30-day and 90day mortality and death at 1 year. Actual date of death and cause of death (1a on death certificate) will be recorded. A detailed summary of the study data collection parameters can be found in Appendix B.

For comparison to ELAP data will be collected directly from NELA and ELLSA databases with no extra data required by each participating site.

\section{Decision-making in NoLAP patients}

For each NoLAP patient, the local team will be asked to select from 5 options who or what specialty was involved in making the decision: patient, surgical, anaesthetic, intensive care or other speciality. If more than one option is selected, the local team will be asked to specify the main two. Next, a list of potential influencing factors will be selected by the local team to improve our understanding of the decision-making process (Appendix B). A patient's decision is defined as either by the patient themselves or next of kin (as determined by clinical team) or patient's wishes already known and documented e.g., living will) and highest level of clinical seniority involved in the decision-making.

\section{Sarcopenia calculation}

Assessment of pre-operative computed tomography (CT) scans within three days of surgical review and laparotomy decision will be performed through psoas muscle mass measurement using whole-slice analysis at the L3 level (manual techniques and semi-automated CT planimetry through the validated software package ImageJ).

For manual measurements, total cross-sectional area of psoas muscles (TPA) will be performed at L3 vertebral level where both transverse processes are maximally in view. Individual psoas muscle outline will be drawn freehand to calculate the TPA, prior to standardisation for patient height to provide the total psoas index [TPI; TPA $\left(\mathrm{mm}^{2}\right) /$ height $\left.\left(\mathrm{m}^{2}\right)\right]$ for each patient. To confirm validation of the technique, intra-and interclass correlation coefficients for TPI and semi-automated techniques will be determined using twenty randomly selected CT scans independently scored by two blinded analysers, with correlation coefficients expected to be at least 0.75 for both techniques.

DICOM images from picture archiving and communication system (PACS) will allow for the measurement of tissue radio-density, including muscle and adipose tissue will be taken, while conversion calculations will be performed to allow for the analysis of all types of CT scan (plain/arterial/portal venous). ${ }^{11,12}$

Calculated values will be stratified by BMI and compared to validated threshold values used for the diagnosis of sarcopenia. ${ }^{13,14}$ Clinical frailty scores will be used as an objective measure of sarcopenia in accordance with the European working group on sarcopenia in older people (EWGSOP statement), with comparisons made with CTdiagnosed sarcopenia through the calculation of correlation coefficients. ${ }^{15}$

Timeline for data collection is given in Table 1.

Table 1: Timetable for data collection.

\begin{tabular}{|ll|}
\hline Period & Date \\
\hline Case identification period & 3 months \\
\hline $\begin{array}{l}\text { Data collection completion } \\
\text { date }\end{array}$ & $\begin{array}{l}\text { 90 days after last case } \\
\text { identified }\end{array}$ \\
\hline Validation completion date & $\begin{array}{l}30 \text { days after completion } \\
\text { date }\end{array}$ \\
\hline
\end{tabular}

\section{Primary outcome}

The primary outcome is to estimate the difference in 90Day mortality between NoLAP and ELAP patient groups.

\section{Secondary outcomes}

Reasons for decision not to operate. Prognostic scores: NELA score, Clinical frailty score, sarcopenia, Surgical diagnosis, Blood markers pre-operatively: lactate, C reactive protein; creatinine and white cell count, Length of critical care stay (ICU and HDU combined). Length of hospital stay, 30-day complications, Discharge destination 30-day and 90-day mortality, Death at 1 year, COVID-19 diagnosis and testing and COVID-19 impact on decision making 


\section{Quality assurance}

This study has been registered with clinical trials, registration number: ISRCTN14556210 (www.clinicaltrials. gov).

The quality of the study has been assessed by the following ways: Steering group meetings: 11/06/19 and 02/07/19, Review by OPSOC, Peer review by professionals with relevant expertise (clinical trialist, statisticians, surgeons and geriatricians). Review by research and development department at NHS Greater Glasgow and Clyde (Sponsor Institution) and Review by Swansea trials unit.

\section{Validation}

Validation will be performed on $25 \%$ of data fields for $10 \%$ of cases. The validated fields will include key demographic and outcome data.

\section{Data management}

A specifically designed online secure electronic database hosted by REDCap (www.project-redcap.org), will be used to enter completed datasets. Local collaborators will be provided with password protected log in details to gain access to the secure database for data entry. All investigators and study site staff will comply with the requirements of the general data protection regulation (GDPR) and data protection act 2018 with regards to the collection, storage, processing and disclosure of personal information and will uphold the act's core principles.

All data uploaded will be anonymised by the local collaborators by having a separate data collection spreadsheet with patient hospital numbers alongside database (REDCap) numbers and the spreadsheet will be stored locally.

The individual site PIs and the ELF2 steering committee will monitor the quality of data entered within REDCap, however there will not be a formal data monitoring committee as this is an observational cohort study with no interventions or changes to patients' normal care pathway. The final dataset will be anonymised and accessed only by the ELF2 steering committee.

\section{Statistical analysis and sample size}

Estimates for the proportion of older patients eligible for but not receiving an emergency laparotomy were determined using data from the largest perspective study to date. ${ }^{7}$ An indicative sample size calculation using an estimate of $32 \%$ suggests 700 patients at $95 \%$ power are required for $3.75 \%$ precision (alpha $=0.05$ ) accounting for a $10 \%$ drop out rate. National data demonstrates 25000 ELAPs are performed each year, suggesting a corresponding figure of 8000 NoLAPs. Therefore, 2000 eligible patients are expected over the 90-day data collection period, demonstrating an achievable sample size.

Multi-level logistic regression will be performed to determine the difference in 90-day mortality and postoperative complications between NoLAP and ELAP patients, adjusted for pre-operative patient factors (including age, gender, ASA and frailty score) and hospital fitted as a random effect to account for unmeasured site variation.

Following analysis, each unit will be offered their own data and a summary of national data, however other individual centres will not be identifiable This will allow comparison to local performance and enable local quality improvement work.

\section{Ethics}

Ethical approval was granted on $20^{\text {th }}$ November 2019 (West Midlands- Solihull research ethics committee (19/WM/0304; IRAS 268511) via the proportionate review service. This study will not need individual patient consent.

\section{Registration}

All PIs at participating units must obtain approval from their local research and development department in conjunction with their relevant national organisation.

The HRA (health research authority) for English sites. The NRSPCC (NHS research Scotland permission coordinating centre) for Scottish sites and the health and care research permissions service for Welsh sites.

In addition, the PI at each site will be individually responsible for ensuring that all members of their team have up to date good clinical practice (GCP) training.

\section{Dissemination}

Data will be reported as a whole cohort, but individual units are invited to ask for individual level data to support local service improvement. The study results will be presented at national and international surgical, anaesthetic and geriatric conferences. Manuscript(s) will be prepared following the close of the study.

\section{DISCUSSION}

The UK NELA has hugely raised the profile of emergency surgical care for patients presenting with an acute abdomen. ${ }^{1}$ This audit programme has driven standards through the development of audit standards throughout the patient's pathway. With relatively high morbidity and mortality risk in emergency surgery, it often common practice for emergency surgical cases to be at local governance meetings. The outcomes for patients presenting with surgical pathology who are managed conservatively are often not reported and 
outcomes for those in whom emergency laparotomy is deemed inappropriate (NoLap) for reasons such as patient choice, co-existing co-morbidity or frailty are even less scrutinised. It is therefore unsurprising that outcomes for patients undergoing emergency laparotomy are widely reported in the published literature whilst only two groups have reported on this NoLap population. The Perth (Australia) emergency laparotomy audit prospectively collected NoLAP numbers as part of analysing ELAP patients and found them to account for only $6 \%$ of the overall population being considered for emergency laparotomy. ${ }^{6}$ This small number limited characterisation of NoLAP patients and more importantly, comparison to ELAP patients. The second study prospectively recorded over three hundred patients requiring ELAP for over 14 months from one UK site. ${ }^{7}$ Patients were included according to the NELA criteria with both NoLAP and ELAP patient groups having the same data recorded. With 'poor fitness' being the commonest reason for NoLAP, this study found that NoLAPs accounted for $32 \%$ of all patients being considered for emergency surgery. In addition, a third of the NoLAP population survived to at least 30 days with normal levels of creatinine and lactate on admission increasing the likelihood of survival. This is the first work to characterise a U.K. NoLAP population, interpretation remains limited by being both single centred and by providing limited information on the decision behind NoLAP.

\section{CONCLUSION}

We aim to apply the findings and challenges from the work in the Perth emergency laparotomy audit and single centre UK NoLap study to definitively define the denominator through this multi-centred cohort study. The data collected in this study will help to inform clinicians, patients and carers in difficult and complex shared decision making to benefit patients. This study will provide valuable information around longer term outcomes for this otherwise relatively unknown patient group.

Funding: No funding sources

Conflict of interest: None declared

Ethical approval: The study was approved by the Institutional Ethics Committee

\section{REFERENCES}

1. NELA. National Emergency Laparotomy Audit. Available at: https://www.nela.org.uk/. Accessed on 3 June 2020.

2. Parmar KL, Pearce L, Farrell I, Hewitt J, Moug S. Influence of frailty in older patients undergoing emergency laparotomy: a UK-based observational study. BMJ Open. 2017;7(e017928):1-6.

3. Clegg A, Young J, Iliff S, Rikkert MO, Rockwood K. Frailty in elderly people. Lancet. 2013;381:75262.

4. Parmar KL, Law J, Carter B, Hewitt J, Boyle JM, Casey $\mathrm{P}$ et al. Frailty in Older Patients Undergoing
Emergency Laparotomy Results From the UK Observational Emergency Laparotomy and Frailty (ELF) Study. Ann Surg. 2019.

5. Carter B, Law J, Hewitt J, Parmar KL, Boyle JM, Casey $\mathrm{P}$ et al. The association between pre-admission frailty and care level at discharge in Older adults undergoing Emergency Laparotomy. BJS - Accept not yet Publ. 2020.

6. Broughton KJ, Aldridge O, Pradhan S, Aitken RJ. The Perth Emergency Laparotomy Audit. ANZ J Surg. 2017;87(11):893-7.

7. Mcilveen EC, Wright E, Shaw M, Edwards J, Vella M, Quasim $\mathrm{T}$ et al. A prospective cohort study characterising patients declined emergency laparotomy: survival in the ' NoLap' population. Anaesthesia. 2019; 75(1):54-62.

8. Charlson ME, Pompei P, Ales KL, MacKenzie CR. A New Method of Classifying Prognostic Comorbidity in Longitudinal Studies: Development and Validation. J Chronic Dis. 1987;40(5):373-83.

9. Rockwood K, Song X, Macknight C, Bergman H, Hogan DB, Mcdowell I et al. A global clinical measure of fitness and frailty in elderly people. CMAJ. 2005;173(5):9-13.

10. Eugene N, Oliver CM, Bassett MG, Poulton TE, Kuryba A, Johnston C et al. Development and internal validation of a novel risk adjustment model for adult patients undergoing emergency laparotomy surgery : the National Emergency Laparotomy Audit risk model. Br J Anaesth. 2018;121(4):739-48.

11. Van Vugt JLA, Van den Braak RRJC, Schippers HJW, Veen KM, Levolger S, De Bruin RWF et al. Contrast-enhancement influences skeletal muscle density, but not skeletal muscle mass , measurements on computed tomography. Clin Nutr. 2017; $1-8$.

12. Rollins KE, Javanmard-emamghissi H, Awwad A, Macdonald IA, Fearon KCH, Lobo DN. Body composition measurement using computed tomography: Does the phase of the scan matter? Nutrition. 2017;41:37-44.

13. Martin L, Birdsell L, Macdonald N, Reiman T, Clandinin MT, Mccargar LJ et al. Cancer Cachexia in the Age of Obesity: Skeletal Muscle Depletion Is a Powerful Prognostic Factor, Independent of Body Mass Index. J Clin Oncol. 2013;31(12):1539-47.

14. Mourtzakis M, Prado CMM, Lieffers JR, Reiman T, Mccargar LJ, Baracos VE. A practical and precise approach to quantification of body composition in cancer patients using computed tomography images acquired during routine care. Appl Physiol Nutr Metab. 2008;33(5):997-1006.

15. Cruz-Jentoft AJ, Bahat G, Bauer J, Boirie Y, Bruyere O, Cederholm T et al. Sarcopenia : revised European consensus on definition and diagnosis. Age Ageing. 2018;48(1):16-31.

Cite this article as: Reeves N, Chandler S, McLennan E, Price A, Boyle J, Knight S, et al. Defining the older patient population that require, but do not undergo emergency laparotomy: an observational cohort study protocol. Int J Clin Trials 2021;8(2):138-44. 


\section{APPENDIX A}

\section{NELA criteria}

\section{NELA inclusion criteria}

- Open, laparoscopic, or laparoscopically-assisted procedures

- Procedures involving the stomach, small or large bowel, or rectum for conditions such as perforation, ischaemia, abdominal abscess, bleeding or obstruction

- Washout/evacuation of intra-peritoneal abscess (unless due to appendicitis or cholecystitis-excluded, see below)

- Washout/evacuation of intra-peritoneal haematoma

- Bowel resection/repair due to incarcerated incisional, umbilical, inguinal and femoral hernias (but not hernia repair without bowel resection/repair). e.g., Large incisional hernia repair with bowel resection

- Bowel resection/repair due to obstructing/incarcerated incisional hernias provided the presentation and findings were acute. This will include large incisional hernia repair with division of adhesions.

- Laparotomy/laparoscopy with inoperable pathology (e.g., peritoneal/hepatic metastases) where the intention was to perform a definitive procedure. This does not include purely diagnostic procedures.

- Laparoscopic/open Adhesiolysis

- Return to theatre for repair of substantial dehiscence of major abdominal wound (i.e., "burst abdomen")

- Any reoperation/return to theatre for complications of elective general/upper GI surgery meeting the criteria above is included. Returns to theatre for complications following non-GI surgery are now excluded (see exclusion criteria below).

\section{NELA exclusion criteria}

- $\quad$ Elective laparotomy/laparoscopy

- Appendicectomy and/or drainage of localised collection unless the procedure is incidental to a non-elective procedure on the GI tract

- Cholecystectomy and/or drainage of localised collection unless the procedure is incidental to a non-elective procedure on the GI tract

- (All surgery involving the appendix or gallbladder, including any surgery relating to complications such as abscess or bile leak is excluded. The only exception to this is if carried out as an incidental procedure to a more major procedure. We acknowledge that there might be extreme cases of peritoneal contamination, but total exclusion avoids subjective judgement calls about severity of contamination.)

- Non-elective hernia repair without bowel resection or division of adhesions

- Minor abdominal wound dehiscence unless this causes bowel complications requiring resection

- Non-elective formation of a colostomy or ileostomy as either a trephine or a laparoscopic procedure (NB: if a midline laparotomy is performed, with the primary procedure being formation of a stoma then this should be included)

- Vascular surgery, including abdominal aortic aneurysm repair

- Caesarean section or obstetric laparotomies

- Gynaecological laparotomy

- Ruptured ectopic pregnancy, or pelvic abscesses due to pelvic inflammatory disease

- Laparotomy/laparoscopy for pathology caused by blunt or penetrating trauma

- All surgery relating to organ transplantation (including returns to theatre for any reason following transplant surgery)

- Surgery relating to sclerosing peritonitis

- $\quad$ Surgery for removal of dialysis catheters

- Laparotomy/laparoscopy for oesophageal pathology

- Laparotomy/laparoscopy for pathology of the spleen, renal tract, kidneys, liver, gall bladder and biliary tree, pancreas or urinary tract

- Returns to theatre for complications (e.g., bowel injury, haematoma, collection) following non-GI surgery are now excluded. i.e., returns to theatre following renal, urological, gynaecological, vascular, hepatic, pancreatic, splenic surgery are excluded. 


\section{APPENDIX B}

\section{CRF}

Table 2: Data collection parameters.

\begin{tabular}{|c|c|c|c|}
\hline \multicolumn{4}{|c|}{ Detailed summary of data collection parameters } \\
\hline Q2 & Age at admission to study (years) & & \\
\hline Q3 & Sex & Male/Female & \\
\hline Q4 & Comorbidities & $\begin{array}{l}\text { CCF Y/N } \\
\text { CVA Y/N } \\
\text { Hemiplegia Y/N } \\
\text { Leukaemia Y/N } \\
\text { Lymphoma Y/N } \\
\text { Mild liver disease Y/N } \\
\text { Severe liver disease Y/N } \\
\text { Solid tumour Y/N } \\
\text { AIDS Y/N } \\
\text { Other: }\end{array}$ & $\begin{array}{l}\text { COPD Y/N } \\
\text { Dementia Y/N } \\
\text { CKD Y/N } \\
\text { DM (complicated) Y/N } \\
\text { DM (uncomplicated) Y/N } \\
\text { IHD Y/N } \\
\text { PVD Y/N } \\
\text { Metastatic tumour Y/N }\end{array}$ \\
\hline Q5 & Polypharmacy ( $\geq 5$ medications) & Yes/No & \\
\hline Q6 & Place of Surgical review (choose most relevant) & $\begin{array}{l}\text { Emergency department } \\
\text { General surgical ward } \\
\text { Another surgical ward } \\
\text { Orthopaedics } \\
\text { Geriatric ward } \\
\text { Acute medical ward } \\
\text { Another medical ward }\end{array}$ & \\
\hline Q7 & Care level prior to admission & $\begin{array}{l}\text { Home (no carers) } \\
\text { Home (with carers) } \\
\text { Residential Home } \\
\text { Nursing home } \\
\text { Intermediate care } \\
\text { Other: }\end{array}$ & \\
\hline Q8 & Frailty score & $1,2,3,4,5,6,7$ & \\
\hline Q9 & NELA score & $-----\%$ & \\
\hline Q10 & Interval between admission and laparotomy (days) & & \\
\hline $\begin{array}{l}\text { Q11a } \\
\text { Q11b } \\
\text { Q11c } \\
\text { Q11d }\end{array}$ & $\begin{array}{l}\text { Pre-decision creatinine } \\
\text { Pre-decision CRP } \\
\text { Pre-decision WCC } \\
\text { Pre-decision Lactate } \\
\text { (all within } 8 \text { hours of decision) }\end{array}$ & & \\
\hline Q11 & Pre-operative clinical diagnosis & & \\
\hline Q12 & CT performed pre-operatively (within 24 hours of decision) & Yes/No & \\
\hline Q13 & CT diagnosis & & \\
\hline Q14 & Psoas muscle mass (TPI) [TPA/ height) & & \\
\hline Q15 & ELAP? & Yes/No & \\
\hline Q16 & Day of decision & $\begin{array}{l}\text { Monday to Thursday } \\
\text { Friday to Sunday }\end{array}$ & \\
\hline Q17 & Time of decision ( 24 hour) & ----:---- & \\
\hline Q18 & Operation performed at laparotomy & & \\
\hline Q19 & Length of stay post-operative or NOLAP decision (days) & & \\
\hline Q20 & Length of critical care stay (ICU and HDU) (days) & & \\
\hline $\begin{array}{l}\text { Q21a } \\
\text { Q21b }\end{array}$ & $\begin{array}{l}\text { Post-operative complication within } 30 \text { days } \\
\text { Grade of complication }\end{array}$ & Yes/No & \\
\hline Q22 & Care level on discharge & $\begin{array}{l}\text { Home (no carers) } \\
\text { Home (with carers) } \\
\text { Residential Home } \\
\text { Nursing home } \\
\text { Intermediate care } \\
\text { Other: }\end{array}$ & \\
\hline Q23 & 30-day mortality & Yes/No & \\
\hline Q24 & 30-day re-admission & Yes/No & \\
\hline Q25 & 90-day mortality & Yes/No & \\
\hline Q26 & 1 year mortality & Yes/No & \\
\hline
\end{tabular}

